\title{
Panorama actual de la taxonomía vegetal en México
}

\author{
FERNANDO CHIANG, PATRICIA DÁVILA \\ Y JOSE LUIS VILLASEÑOR
}

Instituto de Biología, UNAM, Departamento de Botánica. Apdo. Postal 70-367. México, 04510, D.F.

\begin{abstract}
Resumen. Se presenta un panorama de la taxonomía vegetal en México. Se hace un análisis de la cantidad de taxónomos vegetales existentes en México en la actualidad, y cómo esta cifra ha ido aumentando en los últimos 35 años, así como han aumentado los grupos taxonómicos en los que se cuenta con especialistas. Se analiza la literatura de tesis para saber cuántas han sido realizadas sobre temas taxonómicos. Se hace una revisión de la literatura para obtener las aportaciones hechas por mexicanos en temas taxonómicos, utilizando metodología feneticista y/o cladista. Se proporcionan algunos puntos de vista sobre la necesidad de un posgrado moderno en taxonomía.
\end{abstract}

Abstract. An overview of plant taxonomy in Mexico is presented. An analysis is made on the number of plant taxonomists in Mexico at present, and how that number has increased in the last 35 years, as well as plant groups counting with specialists. The thesis literature was searched in order to find out the theses that have dealt with taxonomic subjects. The taxonomic literature was revised in order to obtain the extent of the contribution of Mexicans using pheneticist and/or cladistic methods. Some points of view about the need for a modern postgraduate program in taxonomy are presented.

\section{INTRODUCCIÓN}

Rzedowski $(1981,1993)$ ha presentado interesantes y detalladas descripciones de sucesos de la botánica mexicana a través del tiempo, desde la publicación del Códice de la Cruz-Badiano y las expediciones de Francisco Hernández y de Martín Sessé y José Mariano Mociño hasta la época presente. Este autor (Rzedowski, 1993) hace en particular una evaluación de lo realizado en nuestro país en los últimos cuarenta años de actividades botánicas. Como conclusiones señala: «Requerimos de una masa crítica mínima de 100 botánicos con capacidad para dedicar al estudio de la Flora de México al menos la mitad de su tiempo. Requerimos que muchos de nuestros taxónomos vayan abordando lo antes posible los grupos tradicionalmente más difíciles y menos conocidos, pues sin su revisión profunda, la Flora siempre estará cojeando».

Estos puntos de vista subrayan la necesidad del trabajo llevado a cabo por los taxónomos, orientado a la elaboración de los trabajos florístico-taxonómicos que permitirán algún día poseer la tan anhelada Flora de México. Sin embargo, no toman en cuenta los tratamientos taxonómicos de índole monográfica o de revisión, que cubren información sistemática para las familias, géneros o complejos de especies. En este ensayo se discute acerca de la contribución de la botánica mexicana en la taxonomía de los diferentes grupos vegetales que conforman la Flora de México.

Para comprender el grado de participación de los botánicos mexicanos en el conocimiento taxonómico de los distintos grupos vegetales, se debería realizar un análisis del número de taxónomos que han llevado o llevan a cabo estudios en taxonomía vegetal, las instituciones de investigación donde se producen tales trabajos, así como las obras (tanto publicaciones como tesis no publicadas) realizadas en la disciplina. En el presente artículo se analizan fundamentalmente tres datos:

1 El número de taxónomos vegetales mexicanos con grado de doctorado que en la actualidad están activamente involucrados en la realización de estudios taxonómicos (incluyendo aquellos que participan en la formación de más recursos humanos).

2 El número de trabajos taxonómicos publicados por mexicanos en los últimos años, en los que se utiliza algún tipo de metodología de análisis e inferencia moderno (fenético o cladístico).

3 El número de tesis en taxonomía vegetal producidas en México durante los últimos años.

Es de esperar que la evaluación de las actividades llevadas a cabo por taxónomos mexicanos respecto a los puntos anteriores, permitirá obtener información que ayude a discutir algunos aspectos del estado actual de la taxonomía vegetal en México. Es de esperar también que con esos datos se puedan señalar algunas pespectivas de esta disciplina en los años venideros.

\section{LOS TAXÓNOMOS MEXICANOS}

Hasta la fecha, nuestro país cuenta con aproximadamente 40 taxónomos vegetales con grado de doctor e interesados en el estudio de grupos vegetales mexicanos. Poco más de la mitad de estos doctores se han preparado en el extranjero, 
principalmente en los Estados Unidos, Francia, Inglaterra y Rusia. Desafortunadamente, y por diferentes razones, nueve de estos doctores ya no trabajan en el país. Más de dos tercios de estos taxónomos se han formado en los últimos quince años (Cuadro 1).

CuAdro 1. Número de taxónomos con doctorado formados en los últimos 35 años.

\begin{tabular}{cc}
\hline Año & Taxónomos \\
\hline $1961-1965$ & 3 \\
$1966-1970$ & 2 \\
$1971-1975$ & 4 \\
$1976-1980$ & 0 \\
$1981-1985$ & 10 \\
$1986-1990$ & 6 \\
$1991-1994$ & 15 \\
TOTAL & 40 \\
\hline
\end{tabular}

Por otra parte, actualmente 10 científicos se encuentran en proceso de obtener su doctorado en taxonomía vegetal, tanto en el extranjero como en instituciones educativas del país. Si tomamos en cuenta el número de 100 taxónomos propuesto por Rzedowski (1993) como la masa crítica mínima, en el próximo lustro apenas estaremos llegando a la mitad de este número.

No se debe ignorar que existe un considerable número de botánicos mexicanos que carecen de doctorado y, no obstante, su contribución al conocimiento de la Flora de México ha sido muy importante. Para fines de este análisis, sin embargo, consideramos que larealización de una monografía o revisión taxonómica de buen nivel, implica en la actualidad contar con un marco teórico y metodológico que por lo general sólo puede adquirirse con estudios doctorales formales.

Aun cuando el número de taxónomos mexicanos no es muy grande, su trabajo ha permitido que se empiecen a consolidar grupos de estudio en familias diversas y complejas. Tal es, por ejemplo, el caso de los grupos de trabajo o especialistas en Agavaceae, Anacardiaceae, Araceae, Asclepiadaceae, Asteraceae, Berberidaceae, Boraginaceae, Brassicaceae, Burseraceae, Cactaceae, Caricaceae, Crassulaceae, Cucurbitaceae, Cyperaceae, Dioscoreaceae, Fabaceae, Hernandiaceae, Iridaceae, Lentibulariaceae, Loranthaceae, Magnoliaceae, Marantaceae, Meliaceae, Nolinaceae, Orchidaceae, Poaceae, Podostemaceae, Potamogetonaceae, Rhamnaceae, Rutaceae, Scrophulariaceae y Solanaceae. También hay un notable avance en el estudio de las plantas acuáticas en general, y ya existen taxónomos mexicanos dedicados al estudio de géneros tan complejos como Atriplex, Croton, Datura, Jatropha, Lycium, Physalis, Pinus o Quercus.

\section{TRABAJOS TAXONÓMICOS}

Tras haber sido enunciada la teoría de la evolución, los taxónomos comenzaron a enfatizar relaciones genealógicas de los grupos en sus sistemas de clasificación. Estas relacio- nes eran ilustradas por medio de «árboles» filéticos o filogenéticos. La creación de los «árboles» implicaba la asignación subjetiva de grupos ancestrales o primitivos y grupos derivados o avanzados con base en estados de carácter seleccionados por el especialista. Las relaciones entre los grupos se definían de manera lineal, partiendo por lo general de los más primitivos hacia los más avanzados. Según una crítica a estos métodos subjetivos de clasificación, a manera de mofa, para los taxónomos: «un buen taxón es aquel definido por un buen taxónomo y un buen taxónomo es aquel que ha estado dedicado mucho tiempo al estudio del taxón».

Al comenzar la segunda mitad de este siglo, se gestaron dos escuelas de pensamiento y análisis de los datos utilizados en las decisiones taxonómicas: la escuela feneticista y la escuela cladista. Ambas proponen métodos formales y rigurosos en taxonomía, supuestamente son objetivas, utilizan gran cantidad de caracteres, y en ambas los datos pueden ser corroborados y los resultados sometidos a prueba para confirmarse o refutarse.

El uso de estas metodologías propuestas ha dado por resultado un amplio registro de publicaciones en las que se toman decisiones taxonómicas utilizando los principios y la filosofía de ambas escuelas. Es por esta razón que en este ensayo tocamos el tema referente a trabajos taxonómicos publicados por mexicanos en los últimos años, que utilizan análisis de tipo fenético y/o cladístico, para ver las tendencias en la adopción de uno de los métodos modernos aplicados a la taxonomía.

Existe una amplia literatura acerca de los aspectos teóricos y conceptuales tanto de fenética como de cladística; igualmente, existe un gran número de publicaciones donde estas metodologías han sido aplicadas. Sin embargo, el impacto de estos nuevos métodos de análisis de los datos taxonómicos en la botánica mexicana ha sido prácticamente nulo. La contribución de la botánica mexicana a este tipo de estudios es muy reducida, como puede comprobarse al revisar la literatura buscando las referencias en estos campos, publicadas por botánicos mexicanos.

Kohlmann (1994) discute el escaso uso que hacen los mexicanos de las herramientas fenéticas en estudios taxonómicos. (Aun cuando no está actualizada hasta la fecha, véase la extensa bibliografía proporcionada por Sneath y Sokal (1973) sobre el uso de técnicas fenéticas en biología). Sólo cita tres referencias de taxónomos mexicanos, y a ellas no se pueden añadir muchas más (por ejemplo, Rieseberg et al., 1991, y Villaseñor y Strother, 1989). Por otra parte, algunas veces se ha recurrido al uso de modelos matemáticos aplicados a biogeografía (por ejemplo, Valdés y Espinoza, 1987).

El panorama acerca del uso que hacen los mexicanos de la metodología cladística no es muy distinto al de la fenética. Realmente es muy bajo el número de publicaciones en las que se utiliza esta metodología de análisis. En el Cuadro 2, se presenta una evaluación del número de trabajos publicados desde 1966 (fecha en que se publicó en inglés el trabajo de Hennig; ver Hennig ,1968) hasta 1993, en la que 
se discute algún aspecto relacionado con la cladística. Las cifras mostradas en el Cuadro 2 distan mucho de ser exhaustivas; sin embargo, brindan una idea general del progreso que se ha tenido en la aplicación de este método en la taxonomía y en su consolidación como una poderosa herramienta de análisis. Los trabajos se diferencian entre los puramente teóricos o conceptuales y aquellos en los que se evalúan datos taxonómicos, ya sea morfológicos y/o anatómicos, o bien basados en evidencias obtenidas con las modernas herramientas de biología molecular.

Cuadro 2. Publicaciones que tratan temas de cladística a partir de la publicación en inglés del trabajo de W. Henning (1968) hasta 1993.

\begin{tabular}{lllll}
\hline Año & $\begin{array}{l}\text { Teóricos o } \\
\text { conceptuales }\end{array}$ & $\begin{array}{l}\text { Basados en } \\
\text { morfología } \\
\text { y/o anatomía }\end{array}$ & $\begin{array}{l}\text { Basados en } \\
\text { biología } \\
\text { molecular }\end{array}$ & Total \\
& &
\end{tabular}

\begin{tabular}{lcccc}
\hline $1966-1970$ & 39 & 8 & 0 & 47 \\
$1971-1975$ & 79 & 18 & 3 & 100 \\
$1976-1980$ & 147 & 33 & 1 & 181 \\
$1981-1985$ & 165 & 37 & 4 & 206 \\
$1986-1990$ & 132 & 92 & 43 & 267 \\
$1991-1993$ & 23 & 54 & 60 & 137 \\
TOTAL & 585 & 242 & 111 & 938 \\
\hline
\end{tabular}

Los botánicos mexicanos han publicado muy pocos trabajos empíricos en los que se utilice la metodología cladística. Entre ellos se pueden citar los de Dávila (1991) donde se hace uso de inferencias morfológicas, o los de Delgado-Salinas et al., (1993) o de Rieseberg et al., (1991) donde se recurre a datos moleculares. Algunas otras referencias se pueden encontrar en trabajos que documentan aspectos teóricos o conceptuales del cladismo, como los de Llorente (1989, 1994), Mishler y De Luna (1991) o Villaseñor y Dávila (1992). También se han publicado ensayos sobre el uso del cladismo en la biogeografía (por ej., Espinosa y Llorente, 1991, 1993; Llorente y Espinosa, 1991). Más recientemente, el Museo de Zoología de la Facultad de Ciencias de la UNAM empezó a editar una serie: Principia Taxonomica, que contiene diversas publicaciones conceptuales relacionadas con las ideas básicas y los principios del cladismo y áreas afines.

\section{TESIS PRODUCIDAS EN TAXONOMÍA}

La realización de tesis en taxonomía vegetal por científicos mexicanos, puede ser otro aspecto importante para evaluar la situación de la taxonomía vegetal en nuestro país. Es bien sabido que muchas tesis tienen información inédita de gran valor. Desgraciadamente, un alto porcentaje de ellas nunca llega a publicarse, con lo que su información permanece inaccesible para el público en general. Por lo tanto, se considera importante tener una idea de la magnitud de este acervo.

La información analizada se basó principalmente en dos fuentes. Una es la recopilación hecha por Butanda y
Wong (1994) acerca de las tesis sobre temas botánicos depositadas en la biblioteca del Herbario Nacional del Instituto de Biología de la UNAM. La recopilación incluye todas las tesis contenidas en el acervo, así como trabajos desde 1885 hasta 1994.

La segunda fuente de información corresponde a la contenida en TESIUNAM, que es una recopilación automatizada de todas las tesis realizadas en la UNAM. Aunque las dos fuentes consultadas no cubren toda la información existente a nivel nacional, los datos obtenidos reflejan en gran medida la tendencia nacional en cuanto a trabajos realizados en taxonomía vegetal.

En el Cuadro 3 se presenta el número de tesis evaluadas y relacionadas con aspectos taxonómicos. El 85.5\% de las tesis corresponden a trabajos para obtener el título de biólogo, el $9.3 \%$ a trabajos para obtener el título de maestro en ciencias, y solamente el $5.2 \%$ corresponden a trabajos de tesis a nivel doctoral.

\section{Cuadro 3. Tesis realizadas en taxonomía vegetal y} temas afines.

\begin{tabular}{lcccc}
\hline Area & Licenciatura & Maestría & Doctorado & Total \\
\hline Taxonomía & 55 & 11 & 10 & 76 \\
Florística & 119 & 1 & 4 & 136 \\
Vegetación & 50 & 0 & 1 & 51 \\
Anatomía & 51 & 2 & 2 & 55 \\
Palinología & 7 & 2 & 0 & 9 \\
Citología & 8 & 0 & 1 & 9 \\
Etnobotánica & 81 & 10 & 0 & 91 \\
Fisiología & 15 & 1 & 2 & 18 \\
Ecología & 100 & 9 & 5 & 114 \\
Fitogeografía & 3 & 0 & 0 & 3 \\
Otros & 362 & 46 & 27 & 435 \\
TOTAL & 851 & 94 & 52 & 997 \\
\hline
\end{tabular}

Las tesis analizadas fueron clasificadas en 11 grupos. El grupo 11 (Otros) incluye las tesis llevadas a cabo en áreas tales como ficología, micología, fitoquímica, genética, paleobotánica, microbiología, difusión de la ciencia, educación y edafología.

Como se observa en el Cuadro 3 , únicamente 76 tesis (7.6\%) corresponden a trabajos taxonómicos. De ellas, la mayoría son trabajos realizados a nivel de licenciatura, con un número más o menos equivalente de tesis de maestría y doctorado.

\section{DISCUSIÓN}

La gran riqueza biológica de México, y en particular sus enormes recursos vegetales, muchos de ellos todavía desconocidos por la ciencia, obliga a que se le estudie y se le conozca lo mejor y más rápidamente posible. Esto es más importante ahora que estamos enfrentando la «crisis de la biodiversidad» (donde muchas especies se están extinguiendo antes de ser descritas y clasificadas por los taxónomos). Esta gran necesidad de conocimiento taxonómico de nuestros recursos naturales hace imperativo que los métodos 
empleados sean efectivamente lo más formales y sólidos posibles, especialmente si se quiere utilizar la información generada por los taxónomos en otros campos de la biología comparada.

Es evidente que los nuevos enfoques en taxonomía proporcionados por la fenética y la cladística aún no se arraigan en la comunidad botánica mexicana. El escaso número de contribuciones en estos campos, comparado con la gran cantidad de publicaciones existentes hasta la fecha, indica que estas metodologías no han sido aprovechadas satisfactoriamente. Sin embargo, en algunas instituciones educativas o de investigación, ya existen grupos de trabajo donde comienzan a aplicarse de manera más sistemática en los estudios taxonómicos. En consecuencia, es de esperar que en los próximos años las contribuciones de botánicos mexicanos en estas áreas se incrementen y enriquezcan.

En algunas instituciones, existe la infraestructura adecuada para llevar a cabo estudios de variabilidad genética empleando técnicas electroforéticas en diversos grupos vegetales. Estudios de tal naturaleza han resultado de gran utilidad taxonómica, al revelar variación genética inter e intraespecífica, evidencia acerca de patrones de hibridación, etc. Otra fuente actual de importante información taxonómica la constituyen los estudios llevados a cabo con el ADN, que recurren a diferentes y eficientes metodos de análisis de su constitución. Será necesario elaborar programas y convenios con los poseedores de esta infraestructura, para incorporar su colaboración con los programas que formarán una nueva generación de taxónomos, aquellos que dominen las técnicas modernas necesarias para tomar mejores decisiones taxonómicas. Resulta imperativa la formación de taxónomos de buen nivel y en un lapso relativamente corto, lo cual puede darse si estos grupos de estudio a los que hicimos referencia, reciben apoyo por parte de sus instituciones y del país en general.

El porcentaje de tesis producidas en el área de taxonomía, hace pensar que no existe un interés o motivación entre los jóvenes mexicanos por dedicarse a esta rama de la biología. $\mathrm{O}$ tal vez los taxónomos no hemos puesto un mejor empeño en motivarlos y entusiasmarlos para continuar en esta disciplina. Es muy probable que el bajo índice de tesis y de personas interesadas en la taxonomía, estén ligados a la falta de un posgrado en esta área, pues simplemente no se está dando una opción de preparación formal. También debemos señalar que, junto con el decremento en el interés por desarrollar investigaciones taxonómicas, ha habido un aumento en el interés por otras disciplinas como la ecología, la biogeografía y la conservación de los recursos naturales. Sin embargo, es bien sabido que estas disciplinas siempre están apoyadas en las evidencias taxonómicas y, por lo tanto, no son excluyentes, sino complementarias.

En conclusión, resulta apremiante unir a la comunidad taxonómica con el fin de presentar a los jóvenes mexicanos el panorama que realmente debería de tener la taxonomía en México, en el que los marcos teóricos y metodológicos sean fuertemente discutidos y argumentados, y en donde se ponga de manifiesto su utilidad como disciplina de apoyo a cualquier otra rama de la biología, además de tener un valor inherente e independiente, en el estudio y ordenamiento de los seres vivos.

\section{LITERATURA CITADA}

Butanda A, Wong A. 1994. Tesis sobre temas botánicos en la biblioteca del Herbario Nacional. Cuadernos del Instituto de Biología 20. Instituto de Biología, UNAM. México.

Dávila AP. 1991. Consideraciones filogenéticas y biogeográficas preliminares del género Sorghastrum (Poaceae: Andropogoneae). Acta Bot. Mex. 14: 59-73.

Delgado-Salinas A, Bruneau A, Doyle JJ. 1993. Chloroplast DNA phylogenetic studies in New World Phaseolinae (Leguminosae: Papilionoideae: Phaseoleae). Syst. Bot. 18: 6-17.

Espinosa O DN, Llorente BJ. 1991. Biogeografía de la vicarianza: historia e introducción a los fundamentos y métodos. En: Llorente B J, edr. Historia de la biogeografia: centros de origen y vicarianza. Ciencias, Servicios Editoriales. Facultad de Ciencias, UNAM. México, 35-96.

Espinosa O DN, Llorente BJ. 1993. Fundamentos de biogeografía filogenética. UNAM-CONABIO. Facultad de Ciencias, UNAM. México.

Hennig W. 1968. Elementos de una sistemática filogenética. Eudeba. Buenos Aires, Argentina.

Kohlmann C, B.1994. Algunos aspectos de la taxonomía numérica y sus usos en México. En: Llorente B J, Luna I, comps. Taxonomía biológica. UNAM-Fondo de Cultura Económica, México, 95116.

Llorente BJ. 1989. Algunas ideas de la teoría sistemática contemporánea: conceptos en cladismo. Ciencias. número Especial 3: 26-39.

Llorente BJ. 1994. Conceptos en cladismo. En: Llorente B J, Luna I, comps. Taxonomía biológica. UNAM-Fondo de Cultura Económica, México, 117-141.

Llorente BJ, Espinosa D. 1991. Síntesis de las controversias en la biogeografía histórica contemporánea. Ciencia 42: 295-312.

Mishler BD, De Luna E. 1991. The use of ontogenetic data in phylogenetic analysis of mosses. Adv. Bryol. 4: 121-167.

Rieseberg LH, Beckstrom-Sternberg SM, Liston A, Arias DM. 1991. Phylogenetic and systematic inferences from chloroplast DNA and isozyme variation in Helianthus sect. Helianthus (Asteraceae). Syst. Bot. 16: 50-76.

Rzedowski J. 1981. Un siglo de la botánica en México. Bol. Soc. Bot. México 40: 1-14.

Rzedowski J. 1993. Reflexiones y experiencias sobre el trabajo florístico en México. Memoria del XXX aniversario del Herbario Nacional Forestal y de la VII Reunión Nacional de Encargados de Herbario, 31 julio - 3 agosto 1988. Pub. Esp. del Instituto de Investigaciones Forestales y Agropecuarias (Méx.) 62: 49-58.

Sneath PHA, Sokal RR. 1973. Numerical taxonomy. W. H. Freeman. San Francisco.

Valdés R J, Espinoza AJ. 1987. Corología de las gramíneas de Coahuila, México. Agraria 3: 109-136.

Villaseñor JL, Dávila P. 1992. Breve introducción a la metodología cladística. Prensas de Ciencias. Facultad de Ciencias, UNAM. México.

Villaseñor JL, Strother JL. 1989. Tuxtla, a new genus for Zexmenia pittieri (Compositae: Heliantheae). Syst. Bot. 14: 529-540. 